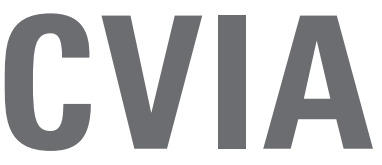

REVIEW ARTICLE

pISSN 2508-707X / eISSN 2508-7088 https://doi.org/10.22468/cvia.2016.00073 CVIA 2017;1(1):4-12

CrossMark \& click for updates

Received: October 28, 2016

Revised: November 10, 2016

Accepted: November 11, 2016

Corresponding author

Charles S White, MD

Department of Diagnostic Radiology,

University of Maryland, 22 S. Greene St.

Baltimore, MD 21201, USA

Tel: 1-410-328-0641

Fax: 1-410-328-0641

E-mail: cwhite@umm.edu

\section{Current Concepts of Vulnerable Plaque on Coronary CT Angiography}

\author{
Seung Min Yoo', Hwa Yeon Lee², Kwang Nam Jin ${ }^{3}$, \\ Eun Ju Chun ${ }^{4}$, Frazier Aletta Ann ${ }^{5}$, Charles S White ${ }^{5}$ \\ 'Department of Diagnostic Radiology, CHA University Bundang Medical Center, Seongnam, Korea \\ ${ }^{2}$ Smile Radiologic Clinic, Seoul, Korea \\ ${ }^{3}$ Department of Radiology, Seoul Metropolitan Government-Seoul National University Boramae \\ Medical Center, Seoul, Korea \\ ${ }^{4}$ Department of Radiology, Seoul National University Bundang Hospital, Seongnam, Korea \\ ${ }^{5}$ Department of Diagnostic Radiology, University of Maryland, Baltimore, MD, USA
}

\begin{abstract}
Most acute cardiac events occur without preceding symptoms and are caused by rupture of vulnerable plaque (thin cap fibroatheroma). Thus, noninvasive identification and modification of vulnerable plaque are of paramount important to reduce cardiac events. In this regard, coronary computed tomography (CT) angiography is an attractive non-invasive imaging tool for identification and evaluation of vulnerable plaque, despite the limited resolution and careful consideration required with the current $C T$ technique. This review article discusses what we know and what remains unclear in the CT evaluation of vulnerable plaque.
\end{abstract}

Key words Coronary artery disease - Atherosclerosis - CT angiography.

\section{INTRODUCTION}

Most acute cardiac events occur without any preceding symptoms. Thus, it is crucial to identify and manage the precursor lesion of an acute cardiac event (i.e., the vulnerable plaque) in asymptomatic patients [1]. Based on postmortem studies, twothirds of acute myocardial infarctions are caused by rupture of vulnerable plaque [2,3]. Pathologic features of vulnerable plaque consist of thin cap fibroatheroma (TCFA) with large plaque burden, a lipid core, positive remodeling, and macrophage infiltration. Widespread use of intravascular ultrasound (IVUS) or IVUS virtual histology (IVUS-VH), optical coherence tomography (OCT), and angioscopy in evaluating vulnerable plaque is limited by the invasive nature of these tools and their inability to examine the entire coronary artery. Notably, acute coronary syndrome (ACS) (i.e., rupture of vulnerable plaque and subsequent intra-arterial thrombosis) is a focal manifestation of systemic disease, but multiple vulnerable plaques are often noted in both the culprit and non-culprit artery [4]. Moreover, a previous study has demonstrated multiple vulnerable plaque ruptures, even in patients with stable angina [5]. Therefore, the ability of computed tomography (CT) to visualize the entire coronary artery system noninvasively is highly attractive. This review will

(c) This is an Open Access article distributed under the terms of the Creative Commons Attribution Non-Commercial License (http://creativecommons.org/licenses/bync/3.0) which permits unrestricted non-commercial use, distribution, and reproduction in any medium, provided the original work is properly cited. discuss CT features of vulnerable plaque, limitations of CT, and future directions.

\section{CT FEATURES OF VULNERABLE PLAQUE}

In contrast to the fibro-calcified plaque (Fig. 1) typical of patients presenting with stable angina, CT features of vulnerable plaque (Figs. 2 and 3) consist of positive remodeling, large plaque burden, small luminal area, low attenuation plaque, the so-call "napkin-ring" sign, and spotty calcifications [5-24].

\section{Positive remodeling}

Most vulnerable plaques are characterized by a large plaque volume. This characteristic renders CT identification of vulnerable plaque more straightforward. The large plaque volume associated with vulnerable plaque is probably caused by disproportional outward growth of the plaque with relatively mild luminal encroachment (i.e., positive remodeling), leading to relatively late development of ischemic symptoms. Thus, because a larger plaque burden exists, a higher degree of positive remodeling is often demonstrated on CT. However, the CT definition of positive remodeling differs from study to study $[1,5-8,11,12,14,21$ 24]. Some studies have used the ratio of vascular area defined as the vascular area at the site of maximal stenosis/vascular area in a normal reference site $[1,12,23,24]$, whereas most studies have employed a simple diameter ratio, probably due to its sim- 


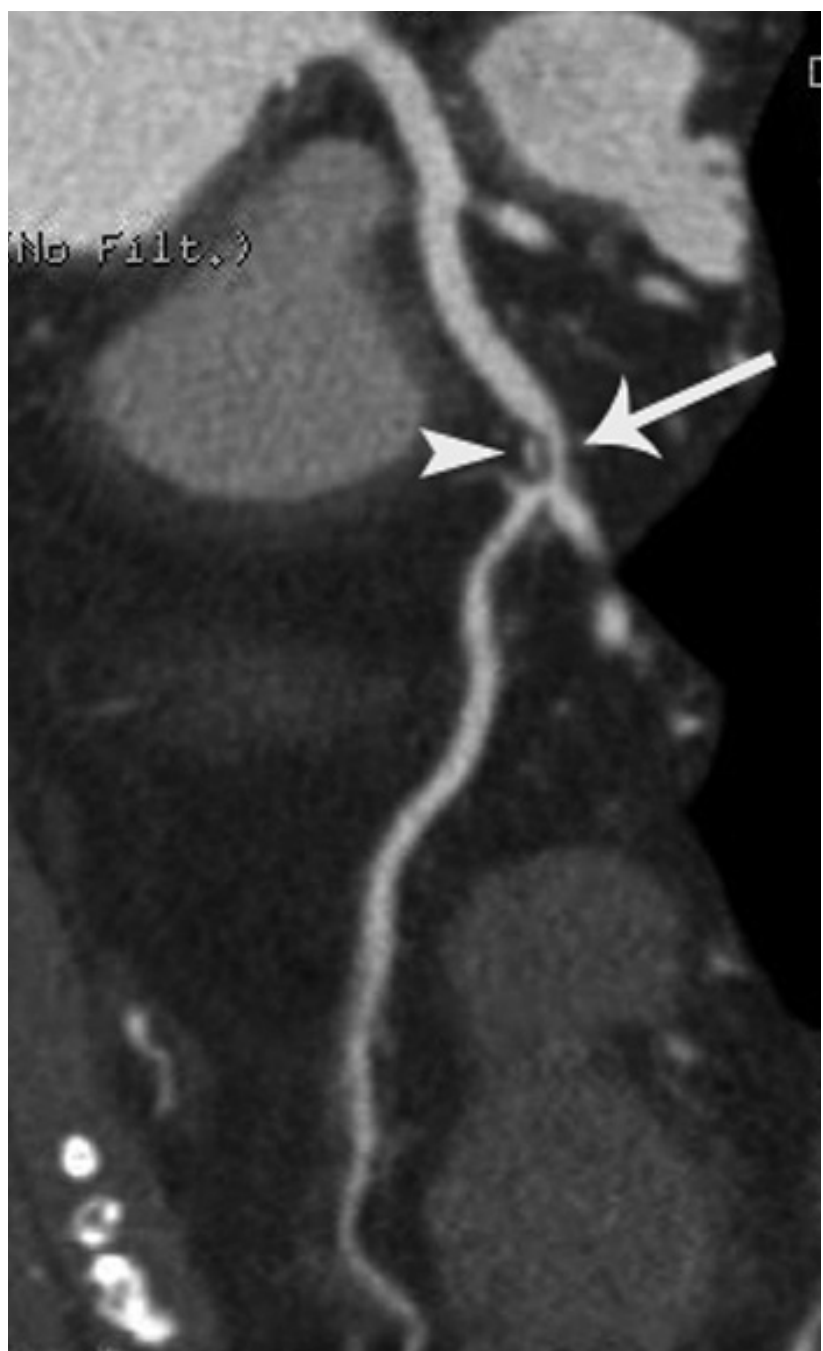

Fig. 1. Typical stable plaque in an 85-year-old woman presenting with atypical chest pain for 3 months. Critical stenosis (arrow) with mainly calcified plaque and low-attenuation plaque (arrowhead) and positive remodeling is noted in the proximal anterior descending coronary artery on a curved multi-planar reformatted image.

plicity $[5-8,11,14,21,22]$. In addition, the cutoff value for designating positive remodeling varies from a ratio of $1.05[5,12,18,24]$ to $1.1[1,6-8,11,14,21,22]$ in these studies. Thus, a standard definition of positive remodeling index is recommended for comparison between studies. Some experts favor measurement of positive remodeling index using vascular area rather than the ratio of lesion diameter because the former approach has less interobserver and intra-observer variability [24]. Although CT permits effective determination of the positive remodeling index, it is sometimes difficult to precisely delineate the outer margin of certain plaques, especially when using a radiation sparing/low dose strategy.

Multiple studies have shown that total plaque burden is a strong predictor of future cardiac event. For example, using an invasive approach, the IVUS-VH study (PROSPECT study) found
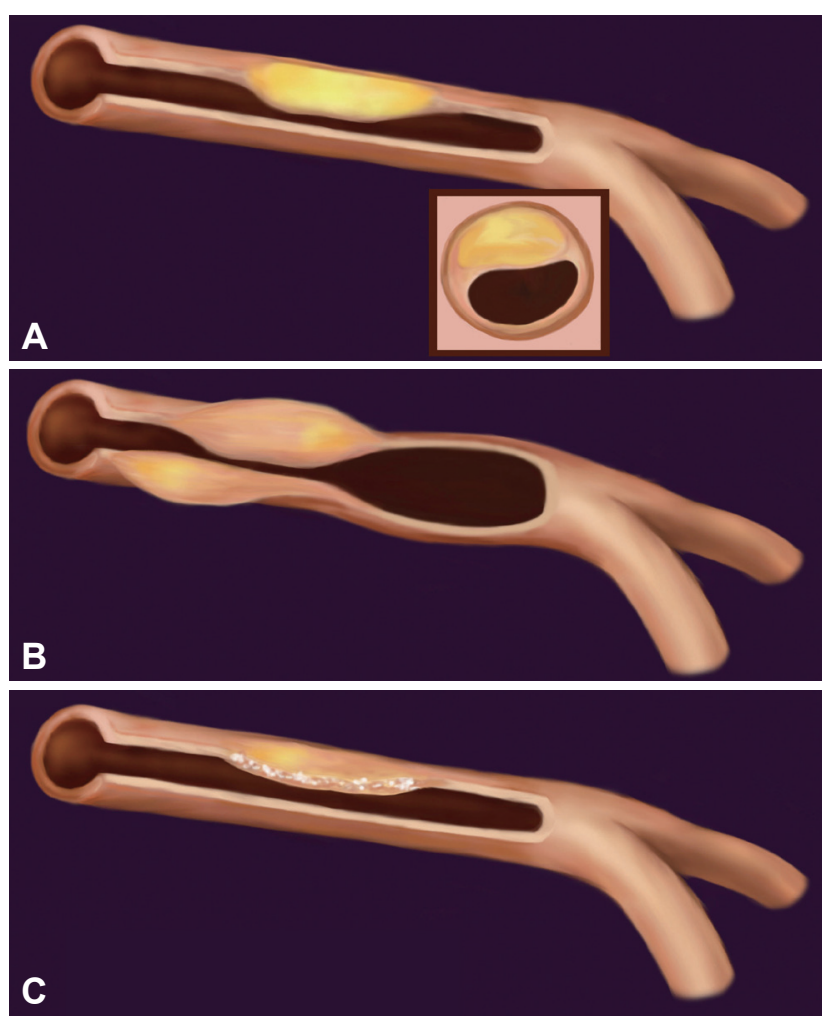

Fig. 2. Several illustrations demonstrating vulnerable plaque. (A) Note a plaque with a large lipid core. The attenuation difference between inner lipid core and outer fibrous plaque in this plaque may be represented by the napkin ring sign on coronary CT angiography. (B) Plaques with positive remodeling. (C) Plaque with spotty calcifications.

that a plaque burden of $\geq 70 \%$ stenosis, minimal luminal area $\leq 4.0 \mathrm{~mm}^{2}$, and TCFA are independent predictors of future cardiac events. A similar strategy might be used on CT to determine plaque burden and minimal luminal area [25]. Furthermore, compared with a simple assessment of the degree of coronary stenosis, measurement of total and low-attenuation plaque burden was superior in predicting ischemia in intermediate stenotic coronary lesions (30-69\%) when using invasive fractional flow reserve as a gold standard [26].

\section{Low-attenuation plaque}

Thrombogenicity of vulnerable plaque is mainly due to elements in the lipid core that contain strong thrombogenic materials. The lipid core of a vulnerable plaque may correspond to a low-attenuation area on CT. However, the definition of low-attenuation plaque also differs from study to study, with Hounsfield units (HU) ranging from 30-60 [5,6-8,19,21,22,24,27], although most studies have used $30 \mathrm{HU}$ as a cutoff [6-8,19,21,22]. This variability is caused by several factors. The CT attenuation value of a particular non-calcified plaque can be different depending on the degree of luminal contrast enhancement, $\mathrm{kV}$ setting, and CT system [28-31]. Although quantitative histogram analy- 

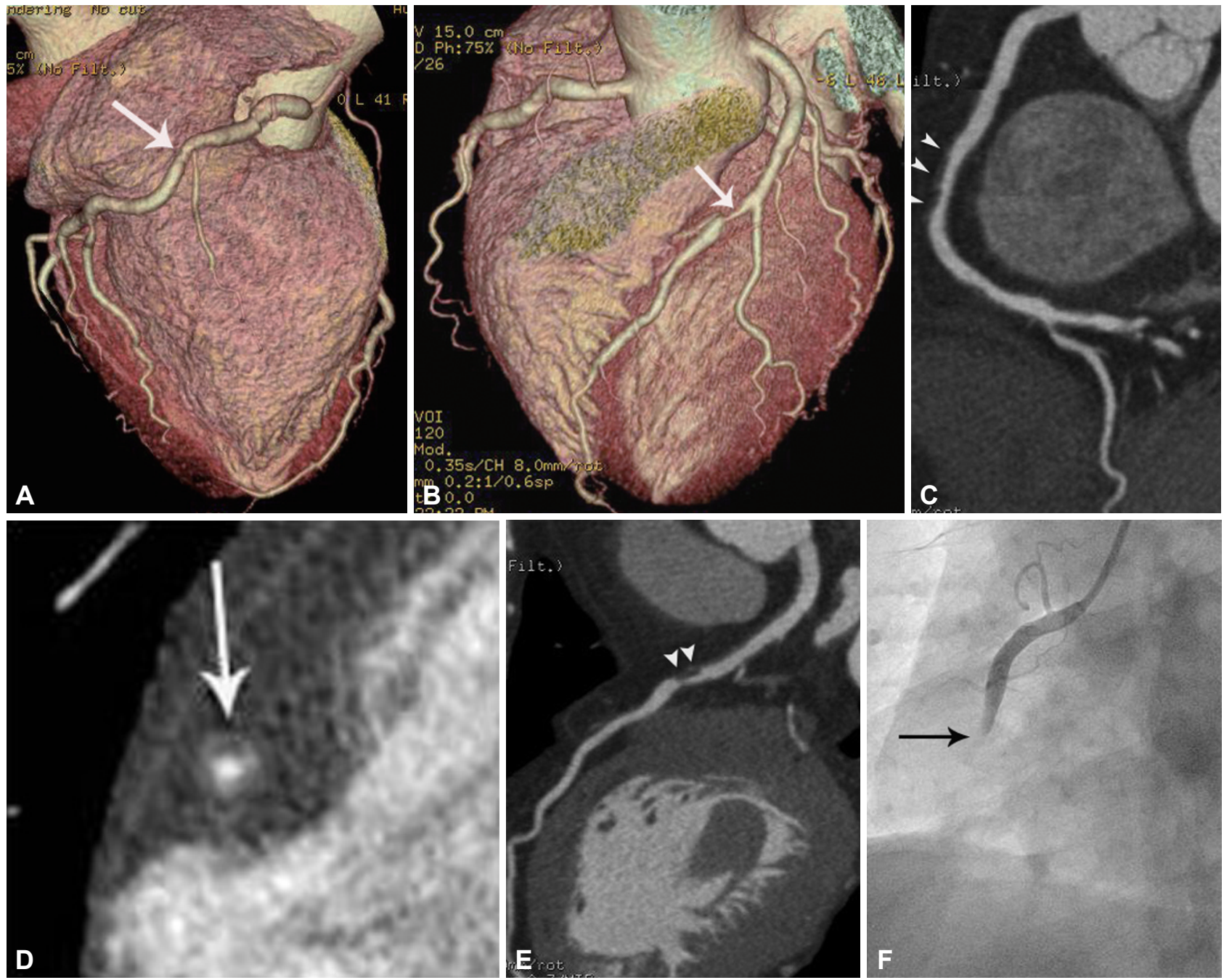

Fig. 3. Asymptomatic 46 -year-old man with multiple vulnerable plaques who subsequently developed ACS. Stenosis of $50-70 \%$ and $>70 \%$ (arrow) is noted on volume-rendered images in the mid-portion of the right coronary (A) and mid-left anterior descending coronary artery (B), respectively. (C) $50-70 \%$ stenosis with noncalcified plaque, positive remodeling, and the napkin ring sign (arrowheads) is noted in the midportion of the right coronary artery on a curved multi-planar reformatted image. Note the napkin ring sign (arrow on D) on a magnified crosssectional multi-planar reformatted image. (E) Critical stenosis (>70\%) with noncalcified plaque and a napkin ring sign, but no definite positive remodeling (arrowheads) is noted in the midportion of the mid-left anterior descending coronary artery on a curved multi-planar reformatted image. Total plaque volume and low-attenuation volume of the former appear to be larger than those of the latter. Positive remodeling is also more prominent in the former. Fifteen months later, the patient developed ACS arising in the mid-portion of the right coronary artery (arrow on F), although the degree of anatomic stenosis was more severe in the plaque in the mid-left anterior descending coronary artery. ACS: acute coronary syndrome.

sis of low-attenuation plaque improves differentiation between lipid-rich and fibrous plaque, use of a single CT density measure has limited value in distinguishing lipid-rich plaque from fibrous plaque [28-30]. Given that the unique technological characteristics of each CT system (e.g., different spatial and temporal resolution, slice thickness, and convolutional kernel) may influence CT attenuation value, a specific cutoff value for low-attenuation plaque should be used for each CT scanner [28]. Furthermore, the approach to measuring CT density of a certain low-attenuation plaque differs from study to study $[1,5,24]$. In one study, the lowest average $\mathrm{HU}$ over multiple regions of interest (ROI) was used [24], whereas another study used the mean value of multiple ROIs [5]. In addition, the size of ROIs differed in these two studies $\left(1 \mathrm{~mm}^{2}\right.$ vs. $\left.1.5 \mathrm{~mm}^{2}\right)[1,24]$. In one previous study, the authors recommended the use of the lowest average value of multiple small ROI (approximately $1 \mathrm{~mm}^{2}$ size at least five different locations) to reduce partial volume averaging [24]. Due to a small risk of radiation-induced cancer, coronary CTA is increasingly performed with a low-radiation exposure strategy (i.e., 80 or $100 \mathrm{kV}$ ). The measured CT density of a lipid core in a low-exposure protocol may differ from that of the standard $120 \mathrm{kV}$ or $135 \mathrm{kV}$ was used in most previous studies. However, relatively few studies have evaluated this issue. 


\section{Napkin ring sign}

Compared with low-attenuation plaque, the napkin ring sign is a more specific CT sign for identification of vulnerable plaque and is an indirect method to detect the presence of TCFA. OCT is the only method that can directly measure the cap thickness of an atheromatous plaque in vivo because the spatial resolution is 10 times higher than that of IVUS $(>100 \mu \mathrm{m})$ [23]. A TCFA is defined as containing a lipid core occupying more than two quadrants of the cross sectional vascular area ( $>180$ degrees) and $<65 \mu \mathrm{m}$ cap thickness on OCT. Thin-cap fibroatheroma is not directly identifiable on CT because its spatial resolution is $0.5 \mathrm{~mm}$ at best. Thus, at least 10 -fold higher spatial resolution would be required to discriminate TCFA from thick cap fibroatheroma or pathologic intimal thickening on CT. However, TCFA can be identified indirectly on CT using the napkin ring sign $[5,12,18]$. The napkin ring sign is defined by inhomogeneous plaque containing a core of lower attenuation material and an outer rim with higher attenuation material. The attenuation value of the outer rim should be less than $130 \mathrm{HU}$ to differentiate it from calcified plaque. The exact pathophysiologic mechanism of the napkin ring sign remains unclear, although multiple hypotheses have been proposed. One hypothesis is that the higher attenuation rim may reflect the attenuation difference between the lipid core and outer fibrous plaque [18]. This hypothesis was derived from an observation that the attenuation value of the outer rim is almost the same in pre-contrast and enhanced CT based on a study using an ex vivo human heart. A second hypothesis is that the high attenuation of the outer rim is caused by enhancement of the vasa vasorum, which can proliferate in circumstances of plaque inflammation. Thus, the napkin ring sign may reflect the degree of active plaque inflammation, which is one of the CT features of plaque vulnerability $[12,18]$. A third hypothesis is that the napkin ring sign depicts central thrombus or hemorrhage with peripheral contrast enhancement, analogous to the CT appearance in venous thrombosis $[12,18]$. A final hypothesis is that the high attenuation outer rim is due to micro-calcification [12].

In one study [5] using angioscopy as a reference standard, the napkin ring sign had the highest positive predictive value (87.5\%) for identifying disrupted plaque consisting of plaque erosion with intact fibrous cap (IFC) or plaque with a ruptured fibrous cap. Moreover, the absence of these three CT features of vulnerable plaque on CT [i.e., positive remodeling (>1.05), lowattenuation plaque $(<40 \mathrm{HU})$, and napkin ring sign] had a relatively high negative predictive value (84.2\%) to exclude disrupted plaques. However, a limitation of the napkin ring sign as a predictor of disrupted plaque is its low sensitivity (43.7\%). This may be due to an inability to identify vulnerable plaque with a small lipid core.

\section{Spotty calcification}

Spotty calcification is defined as calcification with length $<3$ $\mathrm{mm}$ and occupying $\leq 90$ degrees of the vessel arc [19]. An alternative definition of spotty calcification uses the concept of dimensions (i.e., $<2 / 3$ the width and length of the vessel) [24]. The significance of spotty calcification is disputed in the CT literature $[1,6,12]$. Some studies have suggested it as a feature of vulnerable plaque $[1,6]$; however, one study showed no difference in the prevalence of spotty calcification between TCFA and nonTCFA groups (36\% vs. 29\%) [12]. A limitation in the CT evaluation of calcified plaque is that microcalcifications cannot be reliably evaluated on current generation CT scanners due to limited special resolution. In addition, superficial small calcifications can be concealed by luminal contrast enhancement [23]. These limitations may be overcome by advances in CT technique.

\section{PROGNOSTIC VALUE OF VULNERABLE PLAQUE ON CT}

A recent study by Motoyama et al. [8] showed that CT-defined vulnerable plaque [positive remodeling $(\geq 1.1)$ and low-attenuation plaque $(\leq 30 \mathrm{HU})]$ are independent predictors of future acute coronary events. In a study of 3158 patients who underwent coronary CT with a mean follow-up of $3.9 \pm 2.4$ years, $51.1 \%$ $(45 / 88)$ of the acute coronary events arose from areas of CTdefined high-risk plaque [i.e., presence of positive remodeling $(\geq 1.1)$ and/or low-attenuation plaque ( $\leq 30 \mathrm{HU})]$. In addition, plaque progression defined as increase in positive remodeling index or stenosis was an independent predictor of ACS in a subgroup of patients $(\mathrm{n}=449)$ who underwent follow-up CT scan. However, the CT follow-up component in the study may be somewhat limited by the fact that the authors did not use volumetric measurements, which are more accurate in determining plaque progression [9]. In addition, the napkin ring sign, a more objective tool to predict TCFA compared with low-attenuation plaque or positive remodeling, was not evaluated [22]. In one study, ACS occurred at a rate of 0.1 events per year per plaque showing the napkin ring sign [22]. The study also suggested that therapeutic intervention with statins, for example, would eliminate only one-half of future cardiac events because the remainder of ACS events occurred in plaques without CT features of vulnerability [10]. A possible explanation is that TCFA containing a relatively small lipid core and/or lower positive remodeling index may not be identified on CT as a high-risk plaque due to the limited spatial resolution of current CT scanners $[5,27]$. Alternatively, the occurrence of ACS not arising from vulnerable plaque as visualized on CT may be due to intrinsic factors such as the dynamic nature of coronary plaque morphology. The latter possibility is supported by evidence that plaque morphology, especially pathologic intimal thickening, TCFA, and thick cap 
fibroatheroma identified on IVUS at one time point, is not fixed but dynamic [31]. Pathologic intimal thickening or thick cap fibroatheroma can evolve into TCFA, and the reverse can also occur. Thus, it might be necessary to perform follow-up CT scanning to identify such dynamic change of plaque morphology.

\section{LIMITATIONS OF CURRENT CT FOR THE EVALUATION OF VULNERABLE PLAQUE}

\section{Which CT-verified vulnerable plaque will develop future cardiac events?}

The presence of CT-verified vulnerable plaque does not necessarily portend the development of a future cardiac event. In a study by Motoyama, there were significant differences in low-attenuation plaque volume, degree of positive remodeling, and total amount of high-risk plaque [2-feature (with positive remodeling and low-attenuation plaque) or 1-feature positive plaque (with either positive remodeling or low-attenuation plaque)] that subsequently developed ACS compared with high-risk plaques that did not [7]. However, the study did not provide data comparing the volumetric characteristics of the plaques with 2-feature positive plaque or the napkin ring sign between patients who developed ACS and those who did not. In addition, some of their CT studies were obtained on 16-slice MDCT with lower temporal resolution, which is more prone to coronary motion blurring and other artifacts, although no segments were excluded from the study. Second, most CT scanning was performed at $135 \mathrm{kV}$ rather than the standard $120 \mathrm{kV}$, leading to different CT density measurements of low-attenuation plaque than might be prevalent in current clinical practice. Thus, it remains unclear which vulnerable plaque identified on CT will ultimately precipitate a cardiac event. It could be inflammation rather than merely plaque morphologic features that is predictive of future events. Alternatively, future cardiac events might be associated with the degree or amount of specific plaque features (i.e., degree of remodeling index, minimal luminal area, volume of total noncalcified or low-attenuation plaque, and the presence of the napkin ring sign or spotty calcification). This determination requires further studies with larger patient cohorts.

Angioscopic study has indicated that silent rupture of vulnerable plaque is frequent [5]. As most ruptured plaques are clinically silent, the probability of developing ACS from a ruptured plaque is quite low. This finding suggests that there are multiple factors related to development of ACS from a ruptured plaque other than the morphologic features of vulnerable plaque.

Vulnerable blood is an important concept that might be related to the development of ACS from ruptured plaque. For example, smoking is associated with a thrombogenic tendency. Vulnerable blood may be measurable using certain elements such as c-reactive protein [32]. The second factor is vulnerable hemo- dynamic flow around a specific plaque [33]. It is known that wall shear stress is closely associated with formation, maturation, and rupture of a vulnerable plaque [33]. A third factor is the size of the lipid core loaded with potent thrombotic materials that may be related to development of ACS. Finally, the underlying degree of luminal stenosis may be a factor in determining which ruptured plaque subsequently leads to ACS [32]. Even a small thrombosis caused by minor rupture of a fibrous cap can lead to complete occlusion of a coronary artery if the underlying coro-
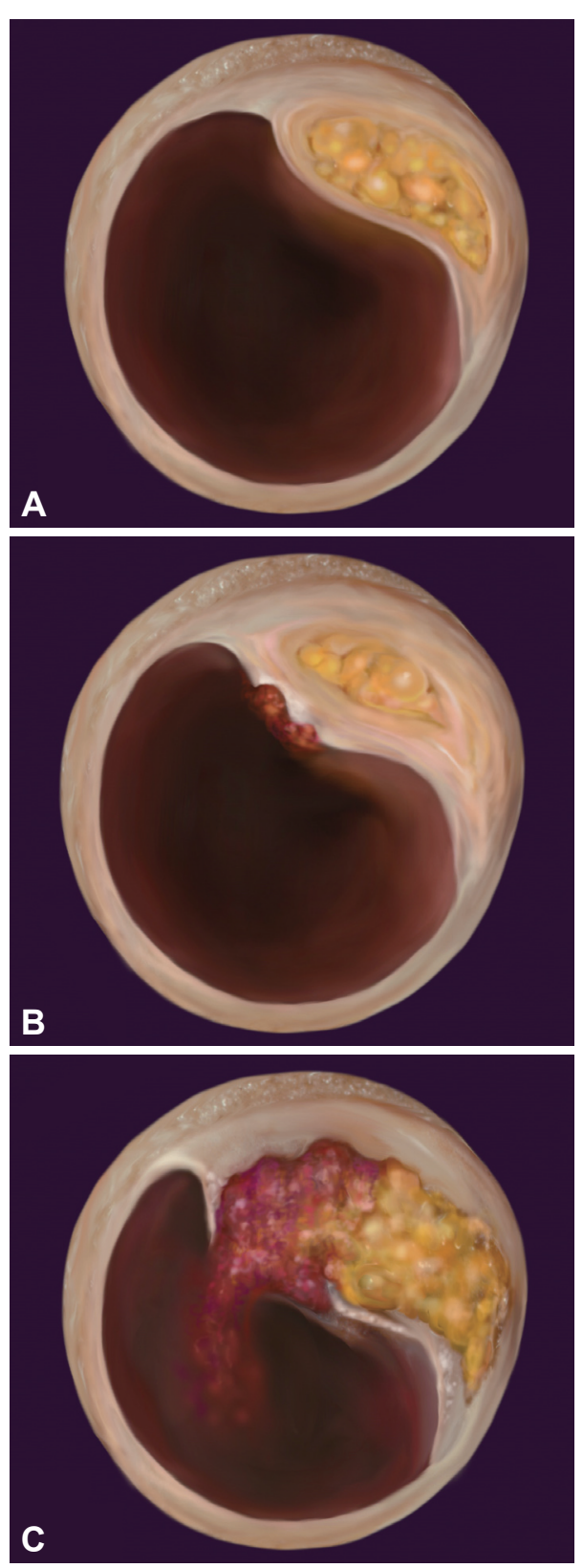

Fig. 4. Representative illustrations showing non-ruptured thin cap fibroatheroma (TCFA) (A), plaque erosion with intact fibrous cap (IFC) (B), and ruptured TCFA (C). Note the increased thickness of the fibrous cap and smaller lipid core of plaque erosion with IFC compared with TCFA. 
nary artery stenosis is critical. Based on these concepts, it might be unwise to treat all vulnerable plaques identified on CT. Rather, a better strategy might be to identify only ruptured vulnerable plaque or to identify factors such as vulnerable blood that link the ruptured vulnerable plaque to an episode of ACS [32].

Activated macrophages produce inflammatory cytokines that
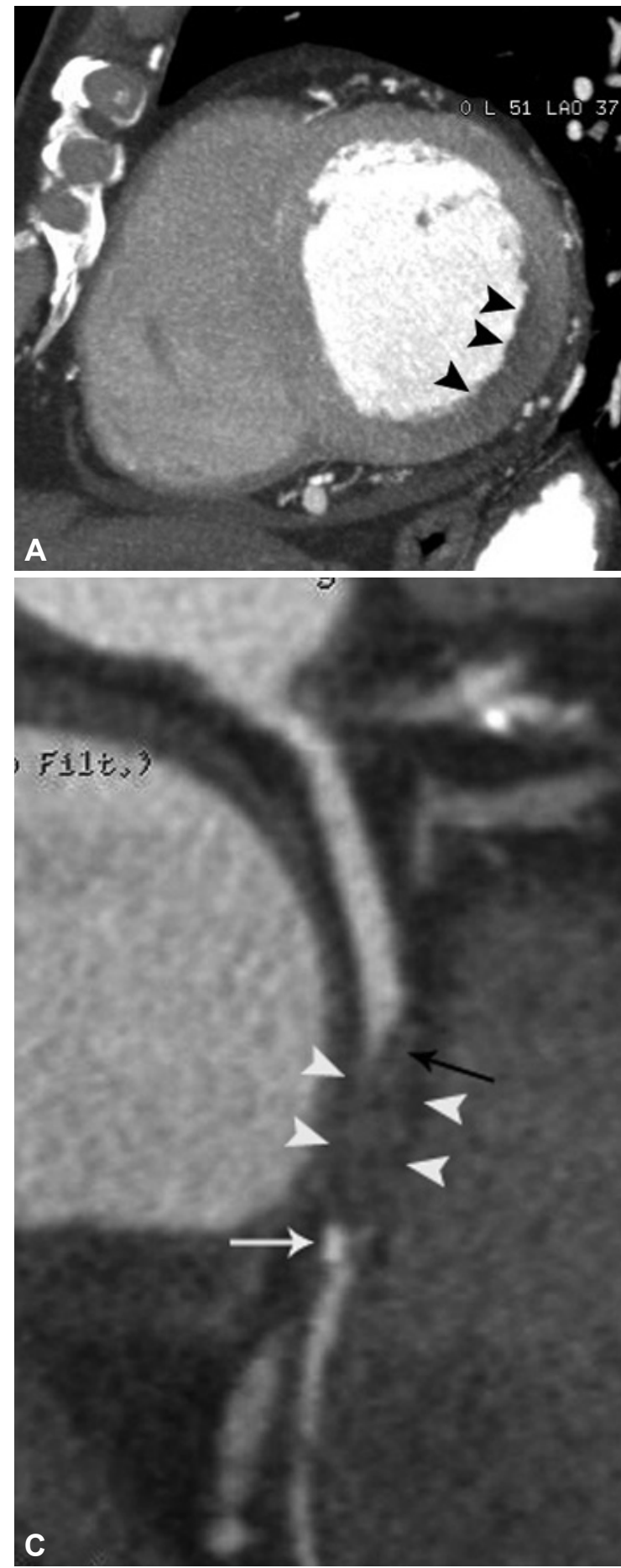
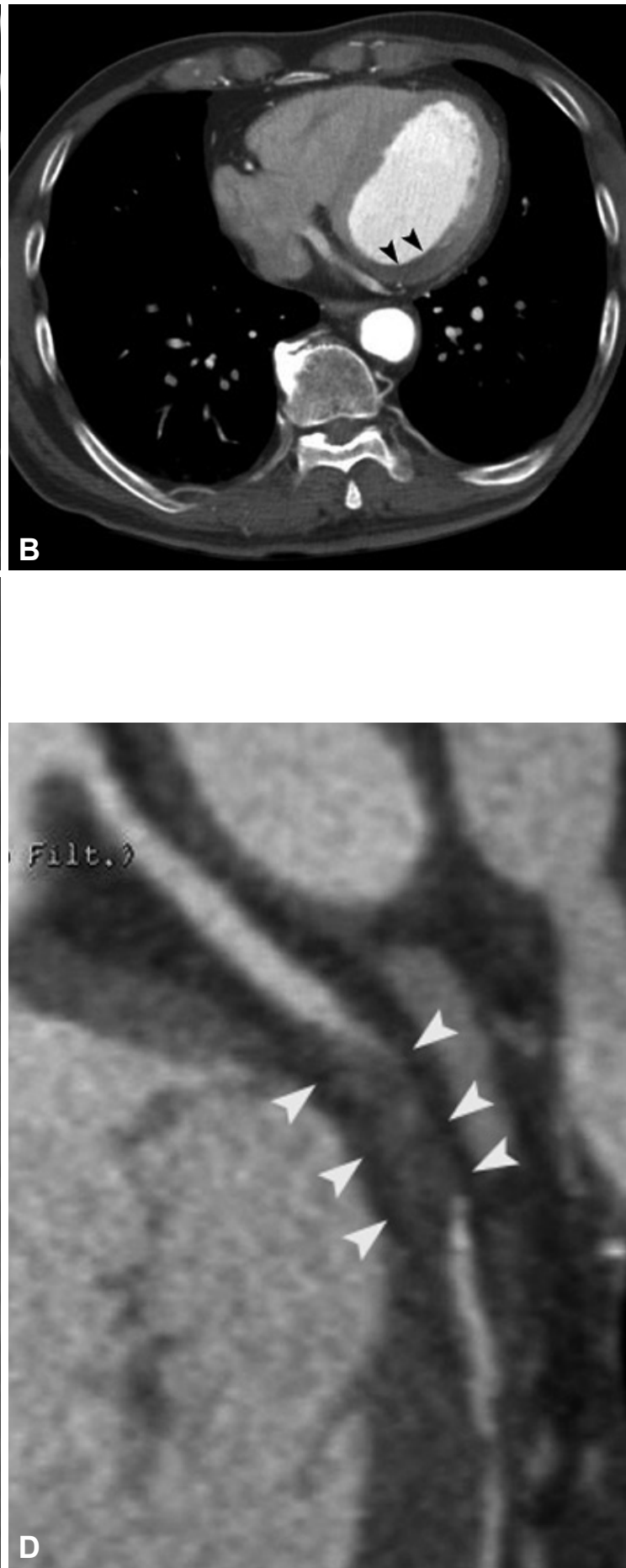

Fig. 5. A culprit lesion on coronary CTA in a 62-year-old-man with acute myocardial infarction. Subendocardial hypoperfusion without myocardial thinning (black arrowheads) is noted in the inferolateral wall on short axis view (A) and axial CT image (B), suggesting acute myocardial infarction. (C) Spotty calcification (small white arrow) and a suspicious small area of low attenuation (small black arrow) are noted on a curved multi-planar reformatted image in the left circumflex artery. Note the obstruction (white arrowheads) in the proximal portion of the distal circumflex coronary artery by thrombus with a CT density of approximately $90 \mathrm{HU}$. (D) A separate curved multiplanar reformatted image shows prominent positive remodeling (>1.5) (white arrowheads) of the culprit plaque. CT: computed tomography, HU: Hounsfield units. 
can induce fibrous cap thinning, rendering specific plaques prone to rupture [2]. Thus, inflammation is closely associated with atherosclerotic plaque progression. In this context, hybrid imaging with PET/CT may be a powerful tool to identify both plaque inflammation and morphology, although this strategy is associated with relatively high radiation exposure and cost, and the spatial resolution of PET is insufficient to analyze small coronary arteries [34,35].

The 2010 ACCF guidelines did not endorse follow-up of the plaque identified on CT [36]. However, one study suggested that follow-up CT can evaluate plaque progression or regression after statin treatment [13]. In this study, plaque volume in 24 patients who took low-dose statins decreased compared with that of controls $(n=8)$ on follow-up CT after one year. In the statin group, the total $\left(92.3 \pm 37.7 \mathrm{~mm}^{3}\right.$ vs. $\left.76.4 \pm 26.5 \mathrm{~mm}^{3}, \mathrm{p}<0.01\right)$ and lowattenuation plaque volumes $\left(4.9 \pm 7.8 \mathrm{~mm}^{3}\right.$ vs. $1.3 \pm 2.3 \mathrm{~mm}^{3}, \mathrm{p}<$ $0.01)$ were significantly decreased. In contrast, there was no significant difference from the control group [13]. However, there are no randomized controlled trials regarding the mortality benefit and cost effectiveness of statin therapy on vulnerable plaque identified on CT. Randomized controlled trials would provide an overview on preventive strategy in asymptomatic patients with CT-verified vulnerable plaque morphology and could suggest an appropriate frequency for follow-up with CT.

\section{Are ACS lesions with an intact fibrous cap visible on CT?}

As noted, the term 'disrupted plaque' includes both ruptured fibrous cap from TCFA and plaque erosion with IFC. Pathologic studies have indicated that plaque erosion with IFC occurs in about one-third of cardiac events [14]. Plaque erosion with IFC is defined as the presence of luminal thrombus without direct contact with deep underlying plaque. The intima of ACS lesions with IFC has endothelial erosion, a rich proteoglycan matrix, and smooth muscle cells. This entity is prevalent in premenopausal women who smoke and accounts for about $80 \%$ of cardiac events in women $<50$ years. Compared with ACS caused by a ruptured fibrous cap, plaque erosion with IFC (Fig. 4) is characterized by less positive remodeling, a smaller lipid core, less spotty calcification, and a lower rate of subsequent cardiac events [37]. In one study, CT features of the plaque erosion with IFC were similar to those of stable plaque (Fig. 2) except for a lower prevalence of large calcification ( $10 \%$ vs. $59 \%, \mathrm{p}=0.001$ ) [14]. In contrast, CT features of plaque erosion with IFC were significantly different from those of ruptured fibrous cap from TCFA. Specifically, the CT prevalence of low-attenuation plaque $(<30$ HU) ( $88 \%$ vs. $40 \%, \mathrm{p}=0.001)$, positive remodeling $(>1.1)(96 \%$ vs. $20 \%, \mathrm{p}=0.001$ ), and spotty calcification $(80 \%$ vs $20 \%, \mathrm{p}=$ 0.001 ) was higher in plaque with a ruptured fibrous cap compared with that found with IFC [14]. Although OCT can be used to differentiate plaque erosion with IFC from plaque with ruptured fibrous cap and stable plaque, no unique features of plaque erosion with IFC have been identified on CT. Thus, it is currently difficult to set up a CT-based strategy to identify patients at high risk for developing ACS who have plaques with IFC. A small vulnerable plaque with a ruptured fibrous cap may not be visible on CT for similar reasons. As spatial resolution on CT improves, these entities may be more easily characterized.

\section{Are CT features of ACS precursor and culprit lesions the same?}

CT features of a culprit lesion after development of ACS may be different than those that occur prior to an initial episode of ACS, at least in theory. This is because CT features of precursor lesions can be altered by thrombosis and rupture (Fig. 5). However, studies by Motoyama have suggested that the characteristics of vulnerable plaque in the pre- and post-cardiac event situations are similar, although these studies did not use a comparative reference standard such as IVUS, angioscopy, or OCT [6-8]. The presence of the napkin ring sign does not necessarily indicate that plaque is disrupted. When disrupted plaque was defined as ruptured TCFA or plaque erosion with IFC and thrombus, at least $12.5 \%$ of plaques with the napkin ring sign were not disrupted [5]. Thus, the difference between disrupted and non-disrupted plaque showing the napkin ring sign requires further investigation. For now, plaque ulceration is the only CT sign that permits differentiation of ruptured from non-ruptured plaque [38].

\section{FUTURE DIRECTIONS}

Although current-generation CT has the potential to evaluate vulnerable plaque, its capabilities are less than ideal. Advancements in CT technology including improved spatial and temporal resolution are mandatory to more precisely evaluate vulnerable plaque. This should be accompanied by strategies to further decrease radiation and contrast use. Other approaches such as the use of dual-energy CT [39], CT/PET hybrid imaging, or the development of new CT contrast agents using nanoparticles may lead to more effective imaging of coronary plaque inflammation. The utility of coronary CTA should be expanded beyond determination of the extent of coronary stenosis to identification and monitoring of vulnerable plaque, with the goal of improving patient outcome.

\section{Conflicts of Interest}

The authors declare that they have no conflict of interest.

\section{REFERENCES}

1. Pflederer T, Marwan M, Schepis T, Ropers D, Seltmann M, Muschiol G, et 
al. Characterization of culprit lesions in acute coronary syndromes using coronary dual-source CT angiography. Atherosclerosis 2010;211:437-444.

2. Libby P. Mechanisms of acute coronary syndromes and their implications for therapy. N Engl J Med 2013;368:2004-2013.

3. Virmani R, Burke AP, Farb A, Kolodgie FD. Pathology of the vulnerable plaque. J Am Coll Cardiol 2006;47(8 Suppl):C13-C18.

4. Rioufol G, Finet G, Ginon I, André-Fouët X, Rossi R, Vialle E, et al. Multiple atherosclerotic plaque rupture in acute coronary syndrome: a threevessel intravascular ultrasound study. Circulation 2002;106:804-808.

5. Nishio M, Ueda Y, Matsuo K, Asai M, Nemoto T, Hirata A, et al. Detection of disrupted plaques by coronary CT: comparison with angioscopy. Heart 2011;97:1397-1402.

6. Motoyama S, Kondo T, Sarai M, Sugiura A, Harigaya H, Sato T, et al. Multislice computed tomographic characteristics of coronary lesions in acute coronary syndromes. J Am Coll Cardiol 2007;50:319-326.

7. Motoyama S, Sarai M, Harigaya $H$, Anno $H$, Inoue $K$, Hara T, et al. Computed tomographic angiography characteristics of atherosclerotic plaques subsequently resulting in acute coronary syndrome. J Am Coll Cardiol 2009;54:49-57.

8. Motoyama S, Ito H, Sarai M, Kondo T, Kawai H, Nagahara Y, et al. Plaque characterization by coronary computed tomography angiography and the likelihood of acute coronary events in mid-term follow-up. J Am Coll Cardiol 2015;66:337-346.

9. Wong DT. Plaque characterization by coronary computed tomography angiography and association with acute coronary syndrome. J Am Coll Cardiol 2016;67:458-459.

10. Braunwald E. Progress in the noninvasive detection of high-risk coronary plaques. J Am Coll Cardiol 2015;66:347-349.

11. Benedek T, Gyöngyösi M, Benedek I. Multislice computed tomographic coronary angiography for quantitative assessment of culprit lesions in acute coronary syndromes. Can J Cardiol 2013;29:364-371.

12. Kashiwagi M, Tanaka A, Kitabata H, Tsujioka H, Kataiwa H, Komukai K, et al. Feasibility of noninvasive assessment of thin-cap fibroatheroma by multidetector computed tomography. JACC Cardiovasc Imaging 2009; 2:1412-1419.

13. Inoue K, Motoyama S, Sarai M, Sato T, Harigaya H, Hara T, et al. Serial coronary CT angiography-verified changes in plaque characteristics as an end point: evaluation of effect of statin intervention. JACC Cardiovasc Imaging 2010;3:691-698.

14. Ozaki Y, Okumura M, Ismail TF, Motoyama S, Naruse H, Hattori K, et al. Coronary CT angiographic characteristics of culprit lesions in acute coronary syndromes not related to plaque rupture as defined by optical coherence tomography and angioscopy. Eur Heart J 2011;32:2814-2823.

15. Motoyama S, Sarai M, Narula J, Ozaki Y. Coronary CT angiography and high-risk plaque morphology. Cardiovasc Interv Ther 2013;28:1-8.

16. Baumann S, Renker M, Meinel FG, Wichmann JL, Fuller SR, Bayer RR 2nd, et al. Computed tomography imaging of coronary artery plaque: characterization and prognosis. Radiol Clin North Am 2015;53:307-315.

17. Alfonso F. Noninvasive detection of vulnerable plaques: are we there yet? J Am Coll Cardiol 2010;55:1163; author reply 1163-1164.

18. Maurovich-Horvat P, Hoffmann U, Vorpahl M, Nakano M, Virmani R, Alkadhi $\mathrm{H}$. The napkin-ring sign: CT signature of high-risk coronary plaques? JACC Cardiovasc Imaging 2010;3:440-444.

19. Shmilovich H, Cheng VY, Tamarappoo BK, Dey D, Nakazato R, Gransar $\mathrm{H}$, et al. Vulnerable plaque features on coronary CT angiography as markers of inducible regional myocardial hypoperfusion from severe coronary artery stenoses. Atherosclerosis 2011;219:588-595.

20. Nakazato R, Shalev A, Doh JH, Koo BK, Dey D, Berman DS, et al. Quantification and characterisation of coronary artery plaque volume and adverse plaque features by coronary computed tomographic angiography: a direct comparison to intravascular ultrasound. Eur Radiol 2013;23:2109-2117.

21. Narula J, Achenbach S. Napkin-ring necrotic cores: defining circumferential extent of necrotic cores in unstable plaques. JACC Cardiovasc Im- aging 2009;2:1436-1438.

22. Otsuka K, Fukuda S, Tanaka A, Nakanishi K, Taguchi H, Yoshikawa J, et al. Napkin-ring sign on coronary CT angiography for the prediction of acute coronary syndrome. JACC Cardiovasc Imaging 2013;6:448-457.

23. Ito T, Terashima M, Kaneda H, Nasu K, Matsuo H, Ehara M, et al. Comparison of in vivo assessment of vulnerable plaque by 64 -slice multislice computed tomography versus optical coherence tomography. Am J Cardiol 2011;107:1270-1277.

24. Kitagawa T, Yamamoto H, Horiguchi J, Ohhashi N, Tadehara F, Shokawa T, et al. Characterization of noncalcified coronary plaques and identification of culprit lesions in patients with acute coronary syndrome by 64-slice computed tomography. JACC Cardiovasc Imaging 2009;2:153-160.

25. Stone GW, Maehara A, Lansky AJ, de Bruyne B, Cristea E, Mintz GS, et al. A prospective natural-history study of coronary atherosclerosis. $\mathrm{N}$ Engl J Med 2011;364:226-235.

26. Diaz-Zamudio M, Dey D, Schuhbaeck A, Nakazato R, Gransar H, Slomka PJ, et al. Automated quantitative plaque burden from coronary CT angiography noninvasively predicts hemodynamic significance by using fractional flow reserve in intermediate coronary lesions. Radiology 2015;276:408415.

27. Cademartiri F, Mollet NR, Runza G, Bruining N, Hamers R, Somers P, et al. Influence of intracoronary attenuation on coronary plaque measurements using multislice computed tomography: observations in an ex vivo model of coronary computed tomography angiography. Eur Radiol 2005; 15:1426-1431.

28. Kristanto W, van Ooijen PM, Jansen-van der Weide MC, Vliegenthart R, Oudkerk M. A meta analysis and hierarchical classification of HU-based atherosclerotic plaque characterization criteria. PLoS One 2013;8: e73460.

29. Schlett CL, Maurovich-Horvat P, Ferencik M, Alkadhi H, Stolzmann P, Scheffel $\mathrm{H}$, et al. Histogram analysis of lipid-core plaques in coronary computed tomographic angiography: ex vivo validation against histology. Invest Radiol 2013;48:646-653.

30. Marwan M, Taher MA, El Meniawy K, Awadallah H, Pflederer T, Schuhbäck A, et al. In vivo CT detection of lipid-rich coronary artery atherosclerotic plaques using quantitative histogram analysis: a head to head comparison with IVUS. Atherosclerosis 2011;215:110-115.

31. Kubo T, Maehara A, Mintz GS, Doi H, Tsujita K, Choi SY, et al. The dynamic nature of coronary artery lesion morphology assessed by serial virtual histology intravascular ultrasound tissue characterization. J Am Coll Cardiol 2010;55:1590-1597.

32. Ueda Y, Ogasawara N, Matsuo K, Hirotani S, Kashiwase K, Hirata A, et al. Acute coronary syndrome: insight from angioscopy. Circ J 2010;74:411-417.

33. Park JB, Choi G, Chun EJ, Kim HJ, Park J, Jung JH, et al. Computational fluid dynamic measures of wall shear stress are related to coronary lesion characteristics. Heart 2016;102:1655-1661.

34. Hyafil F, Cornily JC, Feig JE, Gordon R, Vucic E, Amirbekian V, et al. Noninvasive detection of macrophages using a nanoparticulate contrast agent for computed tomography. Nat Med 2007;13:636-641.

35. Tawakol A, Finn AV. Imaging inflammatory changes in atherosclerosis multimodal imaging hitting stride. JACC Cardiovasc Imaging 2011;4:11191122.

36. Taylor AJ, Cerqueira M, Hodgson JM, Mark D, Min J, O'Gara P, et al. ACCF/SCCT/ACR/AHA/ASE/ASNC/NASCI/SCAI/SCMR 2010 appropriate use criteria for cardiac computed tomography. A report of the American College of Cardiology Foundation Appropriate Use Criteria Task Force, the Society of Cardiovascular Computed Tomography, the American College of Radiology, the American Heart Association, the American Society of Echocardiography, the American Society of Nuclear Cardiology, the North American Society for Cardiovascular Imaging, the Society for Cardiovascular Angiography and Interventions, and the Society for Cardiovascular Magnetic Resonance. J Am Coll Cardiol 2010;56:1864-1894.

37. Yonetsu T, Lee T, Murai T, Suzuki M, Matsumura A, Hashimoto Y, et al. Plaque morphologies and the clinical prognosis of acute coronary syn- 
drome caused by lesions with intact fibrous cap diagnosed by optical coherence tomography. Int J Cardiol 2016;203:766-774.

38. Tanaka A, Shimada K, Yoshida K, Jissyo S, Tanaka H, Sakamoto M, et al. Non-invasive assessment of plaque rupture by 64 -slice multidetector computed tomography--comparison with intravascular ultrasound. Circ
J 2008;72:1276-1281.

39. Jin KN, De Cecco CN, Caruso D, Tesche C, Spandorfer A, Varga-Szemes A, et al. Myocardial perfusion imaging with dual energy CT. Eur J Radiol 2016;85:1914-1921. 